\title{
Solitary Rectal Ulcer Syndrome in Children and Adolescents
}

\author{
Emily Rothbaum Perito, M.D. ${ }^{1}$, Elizabeth Mileti, D.O. ${ }^{1}$, Deepal H. Dalal, B.S. ${ }^{1}$, Soo-Jin Cho, \\ M.D., Ph.D. ${ }^{2}$, Linda D. Ferrell, M.D. ${ }^{2}$, Marjorie McCracken, M.D.,Ph.D. ${ }^{1}$, and Melvin B \\ Heyman, M.D., M.P.H. ${ }^{1}$ \\ ${ }^{1}$ Department of Pediatrics, University of California, San Francisco \\ ${ }^{2}$ Department of Pathology, University of California, San Francisco
}

\begin{abstract}
Objectives-To describe the presenting symptoms, endoscopic and histologic findings, and clinical courses of pediatric patients diagnosed with solitary rectal ulcer syndrome (SRUS).

Methods-We describe 15 cases of SRUS diagnosed at our institution over a 13-year period. Cases were identified by review of a pathology database and chart review and confirmed by review of biopsies. Data were collected by retrospective chart review.

Results-Presenting symptoms were consistent but non-specific, most commonly including blood in stools, diarrhea alternating with constipation, and abdominal/perianal pain. Fourteen of 15 patients had normal hemoglobin/hematocrit, ESR, and albumin at diagnosis. Endoscopic findings, all limited to the distal rectum, ranged from erythema to ulceration and polypoid lesions. Histology revealed characteristic findings. Stool softeners and mesalamine suppositories improved symptoms, but relapse was common.
\end{abstract}

Conclusions-SRUS in children presents with non-specific symptoms and endoscopic findings. Clinical suspicion is required, and diagnosis requires histologic confirmation. Response to current treatments is variable.

\section{Keywords}

solitary rectal ulcer syndrome; rectal bleeding; pediatrics; constipation; rectum; prolapse

\section{Introduction}

Solitary rectal ulcer syndrome (SRUS) is a rare condition most commonly characterized by rectal pain and bleeding. It can be accompanied by diarrhea or constipation, tenesmus, and rectal prolapse ${ }^{1,2}$. Given its non-specific symptoms, it is often difficult to diagnose, particularly in children. The incidence of SRUS has been estimated at 1 in 100,000 adults, with only a few reported pediatric cases ${ }^{1,3}$. In the largest case series of children to date, 12 of $256(5 \%)$ Iranian children with rectal bleeding and straining at the time of defecation

Correspondence to: Emily Rothbaum Perito, MD, Division of Pediatric Gastroenterology, Hepatology and Nutrition, University of California, 500 Parnassus Ave, MU 408-East, Box 0136, San Francisco, CA 94143-0136, Phone: 415-476-5892, Fax: 415-476-1343, mheyman@peds.ucsf.edu.

No authors have any conflicts of interest related to this report.

This is a PDF file of an unedited manuscript that has been accepted for publication. As a service to our customers we are providing this early version of the manuscript. The manuscript will undergo copyediting, typesetting, and review of the resulting proof before it is published in its final citable form. Please note that during the production process errors may be discovered which could affect the content, and all legal disclaimers that apply to the journal pertain. 
were diagnosed with SRUS ${ }^{3}$. Given that the histological and endoscopic appearance mimics other disorders of the rectum, diagnosis of SRUS can be difficult ${ }^{1,4}$.

We describe the range of presenting symptoms and endoscopic and histologic findings that should raise a clinician's awareness for pediatric patients warranting further assessment for SRUS.

\section{Methods}

All pediatric patients diagnosed with SRUS at the University of California, San Francisco Benioff Children's Hospital (UCSF) and an affiliated practice, Pediatric Gastroenterology Associates, were retrospectively reviewed. Cases were identified by searching medical records for ICD9 code 569.41 and the Department of Pathology's biopsy database using "solitary (rectal) ulcer" and "mucosal prolapse" as search terms. Children less than 21 years old at diagnosis with biopsy proven cases of SRUS between 1997 and 2009 were included.

All slides (H\&E) were reviewed by a pathologist (SJC) to confirm the SRUS diagnosis. SRUS was defined by characteristic changes on rectal biopsy including smooth muscle hyperplasia in the lamina propria, hyperplasia of the muscularis mucosae, surface ulceration, architectural distortion (misshapen crypts), and ectasia of superficial capillaries. Patients whose biopsies were not confirmed as SRUS were excluded. Clinical records were then reviewed to identify presenting symptoms, endoscopic findings, laboratory values, treatments and outcomes. Given the small number of patients in our series, we presented all descriptive statistics as median and interquartile range (IQR).

\section{Results \\ Demographics (Table 1)}

Fifteen pediatric patients from our database were diagnosed with SRUS between 1997 and 2009. Twelve were boys, and nine (60\%) were Caucasian. Median age at diagnosis was 13.9 (IQR 9.8-15.6) years.

\section{Clinical Presentation (Table 1)}

Common presenting symptoms included rectal bleeding, alternating diarrhea and constipation, abdominal pain, and perianal pain with defecation. Nine of $15(60 \%)$ complained of cramping abdominal pain, nine (60\%) had diarrhea, and $11(73 \%)$ had blood in stool. Median duration of symptoms prior to diagnosis was 3.2 (IQR 1.2-5.5) years. Two patients (13\%) were diagnosed with inflammatory bowel disease (IBD) prior to diagnosis with SRUS.

\section{Lab Values}

Laboratory data were collected from the time of initial visit to UCSF. Despite rectal bleeding in $73 \%$ of the patients, none were anemic. Median hemoglobin value was 13.1 (IQR 12.6-14.0) $\mathrm{g} / \mathrm{dL}$. All nine patients with measured serum albumin levels had normal values (4.2, IQR 4.0-4.5 g/dL). Erythrocyte sedimentation rate (ESR) was normal (4.0, IQR $3.5-5 \mathrm{~mm} / \mathrm{h}$ ) in all but one patient, whose ESR was $17 \mathrm{~mm} / \mathrm{h}$. This patient was subsequently diagnosed with HLA-B27 spondyloarthropathy, although endoscopy, colonoscopy, and abdominal imaging showed no evidence of IBD, and biopsies were consistent with SRUS.

\section{Other Diagnoses and Family History}

Of the two patients diagnosed with IBD, one patient had ulcerative colitis in conjunction with a history of autoimmune thyroiditis and autoimmune hepatitis. The other patient had a 
long-standing history of ulcerative colitis prior to the SRUS diagnosis. Both patients first presented with gastrointestinal symptoms, including loose bloody stools and abdominal pain, between 7 to 8 years of age. Both were initially diagnosed with IBD and then subsequently diagnosed with SRUS ( 2.5 and 7 years after presentation, respectively). Both had histologic findings consistent with SRUS in the distal rectum in addition to more proximal inflammation.

None of the 15 patients had a family history of SRUS. Neither of the patients diagnosed with IBD had a family history of IBD, but three of the other patients with SRUS (20\%) had a family history of IBD.

\section{Endoscopic Findings}

Endoscopic findings varied widely. All patients had endoscopic abnormalities in the distal rectum, mostly limited to within $10 \mathrm{~cm}$ of the anal verge. (Figure 1) Of the 10 patients with endoscopic reports available, 8 had visible erythema or inflammation. Four patients had polyps or polypoid lesions.

Patient characteristics are detailed in Table 2.

\section{Histologic Findings (Table 3)}

All patients (15) had at least one rectal biopsy with histologic findings consistent with SRUS or mucosal prolapse. Multiple biopsies were available for review for 6 patients, with histologic features of SRUS seen in multiple biopsies for 4 of these patients. Common histologic findings included muscularization of the lamina propria with strands or bundles of smooth muscle extending between crypts, thickening or hyperplasia of the muscularis mucosae, surface ulceration with inflammation, focal hyperplastic changes in the epithelium, misshapen crypts, and vascular ectasia/congestion, which have all been described in previous reports of SRUS or mucosal prolapse $\mathrm{e}^{5-10}$. (Figures 2, 3) A mixed inflammatory infiltrate was often seen in areas of ulceration, but cryptitis or crypt abscesses and chronic changes characteristic of inflammatory bowel disease were not seen in most biopsies. In the 2 patients with a concurrent diagnosis of IBD, one of the patients had a single biopsy available for review which showed features of SRUS without features of IBD. Five biopsies were available for review for the other patient with IBD, one of which showed features of SRUS without features of IBD, 1 of which showed features of an inflammatory polyp, and 2 of which showed overlapping features (particularly surface ulceration with granulation tissue and dense inflammation). In addition, while reactive epithelial atypia was seen in biopsies of 3 of the patients, none of the biopsies had definitive evidence of dysplasia or adenocarcinoma.

\section{Treatment}

The thirteen patients without IBD were treated with laxatives, stool softeners, and mesalamine suppositories or enemas. Four underwent polypectomy at diagnosis. One patient required proctectomy for recurrent symptomatic polyps. The two patients with IBD were treated with stool softeners, oral or rectal aminosalicylates, oral or rectal corticosteroids, and antibiotics throughout the course of their follow-up. Several of the patients were lost to follow-up after initial diagnosis and treatment, precluding assessment of their response to treatment. In those patients who did have long-term follow-up, 6 of 9 responded to treatment, but at least $50 \%$ had recurrent symptoms, particularly after medication adherence lapsed. 


\section{Discussion}

Our review of pediatric patients at our institution revealed 15 cases of SRUS over a 13-year period. Presenting symptoms were consistent but non-specific. Delay in diagnosis was common, likely because of the non-specific symptoms and rarity of SRUS in the pediatric population. Our cases had a median 3.2 year delay between symptom onset and diagnosis. This is similar to the delay in diagnosis seen in pediatric IBD patients, who also often present with similar symptoms ${ }^{11}$. Treatment led to clinical improvement in most patients, but symptoms often recurred with poor medication adherence.

Our series confirms that SRUS is a misnomer since not all lesions are solitary nor ulcers. Previous reports support this characterization ${ }^{4,12}$. SRUS has also been referred to as mucosal prolapse to avoid this confusion ${ }^{10}$. Endoscopically, the distal rectal mucosa typically appears erythematous, and lesions can be ulcerative or polypoid ${ }^{3,13}$. Differential diagnosis in children includes juvenile polyps, infections, IBD, sexual abuse, or rectal digitations. Because our case search strategy relied on cases confirmed by pathology, we were not able to include cases suspected to be SRUS that did not have pathologic confirmation or were diagnosed as other disorders on histology.

Given the lack of symptom specificity and endoscopic findings seen in our cases, this series supports the necessity for histological diagnosis. The histologic changes are similar to those seen in adults. Characteristic findings include muscularization of the lamina propria, hyperplastic muscularis mucosa, and distortion of crypt architecture ${ }^{9,10,14}$. SRUS is distinguished from IBD by scarring of the lamina propria with only a relatively mild inflammatory infiltrate, as well as the accompanying muscular hyperplasia ${ }^{14}$. Our patients without coexistent IBD had findings limited to the distal rectum.

Two of our cases, however, illustrate that SRUS and IBD can coexist in children and adolescents. Previous case reports in the adult literature have also noted concurrent SRUS and IBD, particularly ulcerative colitis (UC) ${ }^{15,16}$. Thus, a diagnosis of IBD should not preclude consideration of SRUS, as management may differ. For those with concurrent SRUS and IBD, stool softeners and/or anti-inflammatory enemas to treat the SRUS may be necessary even when systemic medications are used to treat the IBD.

Since SRUS and IBD can present with identical symptoms-and have similar endoscopic features-SRUS can also be misdiagnosed as IBD. Misdiagnosis is concerning because the oral or intravenous immunosuppressive medications used for ulcerative colitis are unlikely to be effective in SRUS. In addition, since patients with long-standing IBD colitis can develop malignant lesions in the colon, SRUS could be misdiagnosed as tumor ${ }^{16}$. Thus, histologic confirmation of the diagnosis is important to guide management. IBD with rectal ulcers could also be mistaken for SRUS; in general, features of systemic disease, such as weight loss, extra-intestinal manifestations of IBD, or elevated serum inflammatory markers, should not be present in isolated SRUS.

While its etiology is unclear, SRUS has been linked to direct mucosal trauma, rectal dysmotility, and ischemia. Strained defecation in some patients may lead to direct ulceration of the mucosa. In others, the anterior rectal mucosa is forced into the anal canal, causing rectal prolapse and mucosal injury ${ }^{4,14}$. Rectal prolapse and high intra-abdominal pressures may contribute to vascular compression, leading to mucosal ischemia ${ }^{1}$. Tenesmus and straining related to IBD colitis have been theorized contributors to the development of SRUS in patients with both disorders.

SRUS is not considered to be invasive or progressive. However it tends to be refractory ${ }^{5}$, as the outcomes of our patients suggest. Common treatments include laxatives, stool softeners, 
and rectal aminosalicylates $1,2,17$. Surgery has also occasionally been used with minimal long-term success, since the lesions often recur if the straining and dysmotility continue. In our patients, stool softeners and mesalamine suppositories were most often used. The small number of patients and variable follow-up prevent us from drawing robust conclusions about treatment efficacy.

Our analysis was limited due to the relatively small sample size and missing data. Multicenter prospective cohort studies would help identify treatment methods and outcome of pediatric patients with SRUS.

\section{Acknowledgments}

Partial support for this work is from NIH Grants DK060617 and DK007762.

\section{References}

1. Keshtgar AS. Solitary rectal ulcer syndrome in children. Eur J Gastroenterol Hepatol. 2008:89-92. [PubMed: 18188026]

2. De la Rubia L, Ruiz Villaespesa A, Cebrero M, et al. Solitary rectal ulcer syndrome in a child. J Pediatr. 1993:733-6. [PubMed: 8496752]

3. Dehghani SM, Haghighat M, Imanieh MH, et al. Solitary rectal ulcer syndrome in children: A prospective study of cases from southern iran. Eur J Gastroenterol Hepatol. 2008:93-5. [PubMed: 18188027]

4. Ertem D, Acar Y, Karaa EK, et al. A rare and often unrecognized cause of hematochezia and tenesmus in childhood: Solitary rectal ulcer syndrome. Pediatrics. 2002:e79. [PubMed: 12456946]

5. Madigan MR, Morson BC. Solitary ulcer of the rectum. Gut. 1969:871-81. [PubMed: 5358578]

6. Warren BF, Dankwa EK, Davies JD. 'Diamond-shaped' crypts and mucosal elastin: Helpful diagnostic features in biopsies of rectal prolapse. Histopathology. 1990:129-34. [PubMed: 2227837]

7. Franzin G, Scarpa A, Dina R, et al. "Transitional” and hyperplastic-metaplastic mucosa occurring in solitary ulcer of the rectum. Histopathology. 1981:527-33. [PubMed: 7286915]

8. Tendler DA, Aboudola S, Zacks JF, et al. Prolapsing mucosal polyps: An underrecognized form of colonic polyp--a clinicopathological study of 15 cases. Am J Gastroenterol. 2002:370-6. [PubMed: 11866275]

9. Chiang JM, Changchien CR, Chen JR. Solitary rectal ulcer syndrome: An endoscopic and histological presentation and literature review. Int J Colorectal Dis. 2006:348-56. [PubMed: 16133006]

10. Singh B, Mortensen NJ, Warren BF. Histopathological mimicry in mucosal prolapse. Histopathology. 2007:97-102. [PubMed: 17204024]

11. Heyman MB, Kirschner BS, Gold BD, et al. Children with early-onset inflammatory bowel disease (IBD): Analysis of a pediatric IBD consortium registry. J Pediatr. 2005:35-40. [PubMed: 15644819]

12. Martin CJ, Parks TG, Biggart JD. Solitary rectal ulcer syndrome in Northern Ireland. Br J Surg. 1981:744-7. 1971-1980. [PubMed: 7284739]

13. du Boulay CE, Fairbrother J, Isaacson PG. Mucosal prolapse syndrome--a unifying concept for solitary ulcer syndrome and related disorders. J Clin Pathol. 1983:1264-1268. [PubMed: 6630576]

14. Sharara AI, Azar C, Amr SS, et al. Solitary rectal ulcer syndrome: Endoscopic spectrum and review of the literature. Gastrointest Endosc. 2005:755-62. [PubMed: 16246692]

15. Arhan M, Onal IK, Ozin Y, et al. Solitary rectal ulcer syndrome in association with ulcerative colitis: A case report. Inflamm Bowel Dis. Feb.2010 :190-1. [PubMed: 19418566]

16. Uza N, Nakase H, Nishimura K, et al. Solitary rectal ulcer syndrome associated with ulcerative colitis. Gastrointest Endosc. Feb.2006 :355-6. [PubMed: 16427960] 
17. Bishop PR, Nowicki MJ, Subramony C, et al. Solitary rectal ulcer: A rare cause of gastrointestinal bleeding in an adolescent with hemophilia A. J Clin Gastroenterol. 2001:72-6. [PubMed: 11418797] 


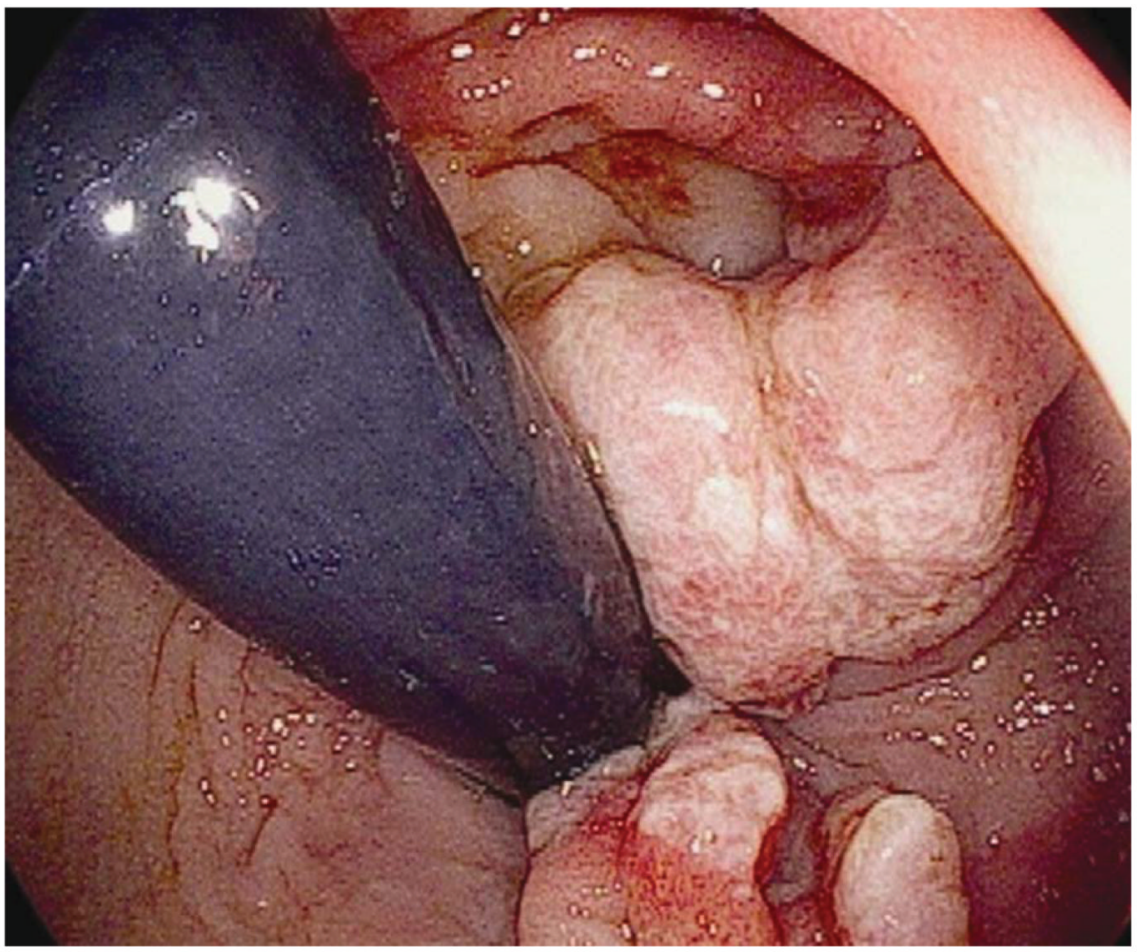

Figure 1.

Endoscopic image of SRUS patient, showing distal rectum on retroflexion. Multiple polypoid lesions surrounded by erythema and inflammatory infiltrates. 


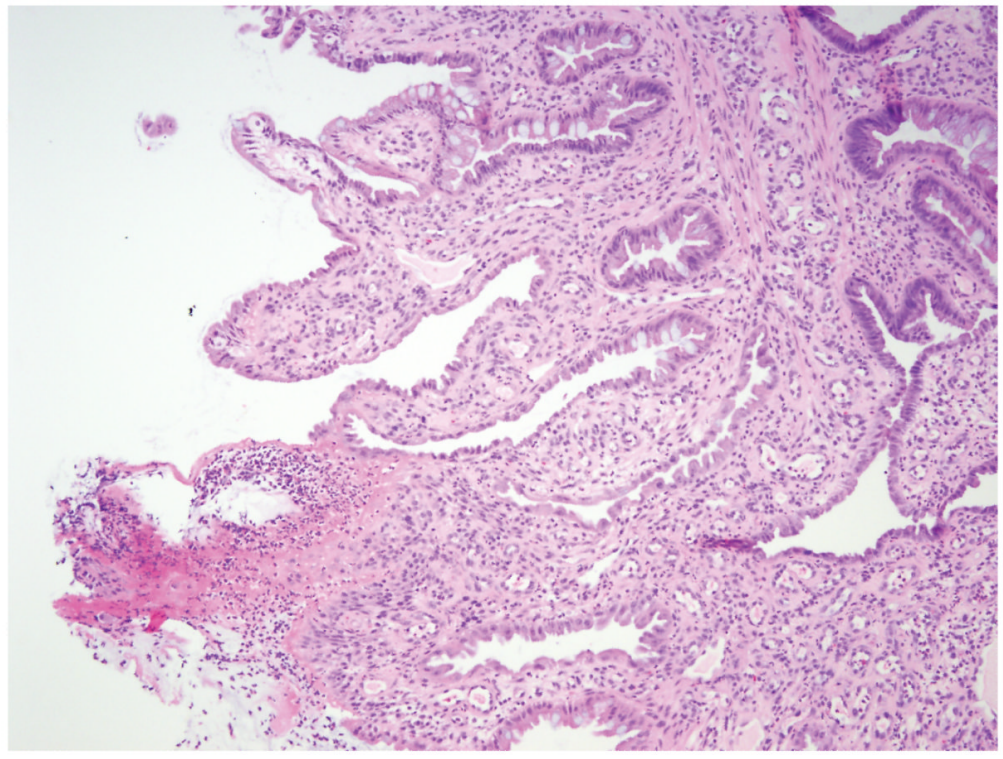

Figure 2.

Rectal biopsy from the SRUS patient whose endoscopy is shown in Figure 1. Histology (H\&E) shows smooth muscle hyperplasia in the lamina propria and surface ulceration with associated acute and chronic inflammatory infiltrates. Crypt architectural changes as well as vascular proliferation and ectasia of superficial mucosa are also present. 


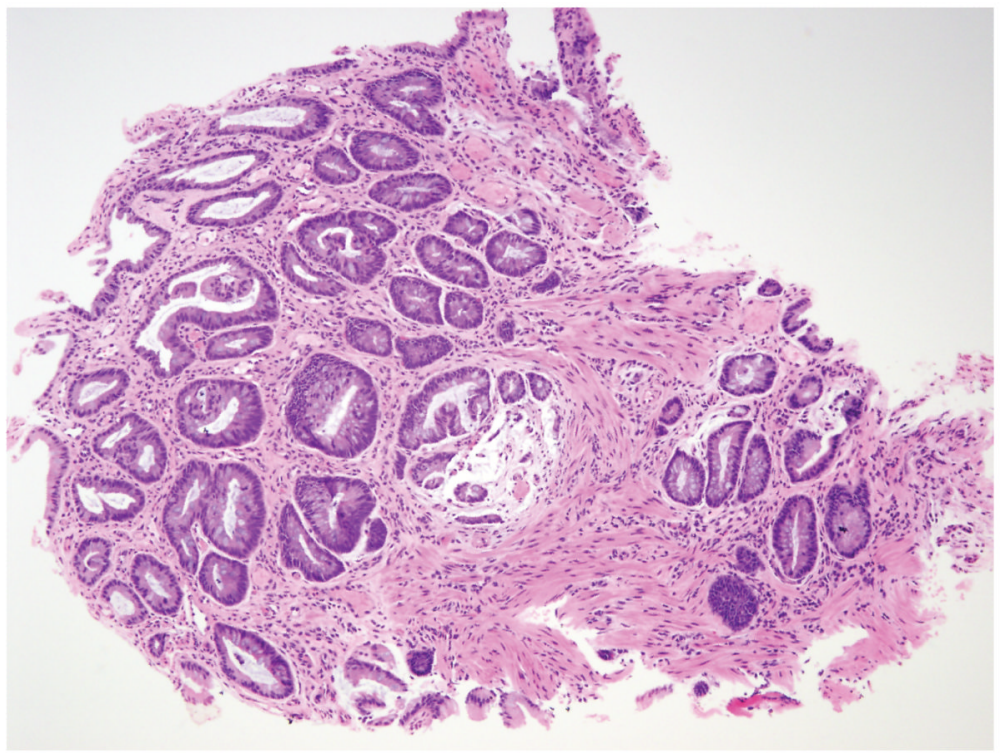

Figure 3.

Rectal biopsy (H\&E) from another SRUS patient showing smooth muscle hyperplasia in the lamina propria and of the muscularis mucosae, as well as crypt architectural distortion with misshapen crypts, mild vascular ectasia of superficial mucosa, and minimal inflammatory infiltrate. 
Table 1

Patient Demographics and Clinical Presentation

\begin{tabular}{|l|c|}
\hline & N \\
\hline Male & 12 \\
\hline Female & 3 \\
\hline Caucasian & 9 \\
\hline Other & 6 \\
\hline Symptoms at Diagnosis & \\
\hline \multicolumn{1}{|c|}{ Blood in stool } & 11 \\
\hline \multicolumn{1}{|c|}{ Abdominal Pain } & 9 \\
\hline \multicolumn{1}{|c|}{ Diarrhea } & 9 \\
\hline \multicolumn{1}{|c|}{ Constipation } & 6 \\
\hline \multicolumn{1}{|c|}{ Perianal pain } & 4 \\
\hline Age at Diagnosis, (median years, IQR) & $13.9(9.8-15.6)$ \\
\hline $\begin{array}{l}\text { Symptomatic Before Diagnosis (median } \\
\text { years, IQR) }\end{array}$ & $3.2(1.2-5.5)$ \\
\hline
\end{tabular}




\begin{tabular}{|c|c|c|c|c|c|c|c|c|c|c|c|c|}
\hline & 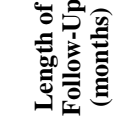 & $m$ & $a$ & $\Phi$ & - & $\approx$ & - & $I$ & q & $n$ & - & $\stackrel{n}{n}$ \\
\hline & 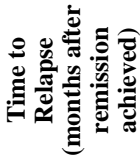 & & $m$ & & & $\mathbb{z}$ & & $\stackrel{n}{n}$ & 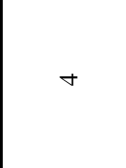 & & $\overleftrightarrow{z}$ & \\
\hline & 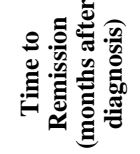 & & in & & & $\simeq$ & & $m$ & $=$ & & $\mathbb{z}$ & \\
\hline & 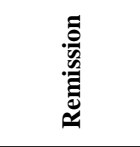 & $\mathbb{z}$ & $\lambda$ & z & $\mathbb{z}$ & $\lambda$ & $\overleftrightarrow{z}$ & $\lambda$ & $>$ & $\mathbb{z}$ & $\succ$ & $\overleftrightarrow{z}$ \\
\hline & : & 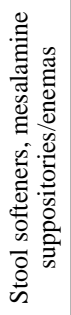 & 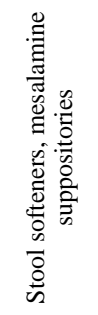 & 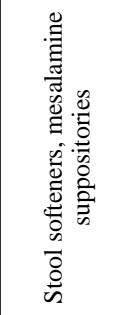 & $\mathbb{z}$ & 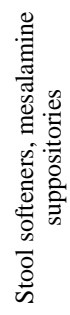 & 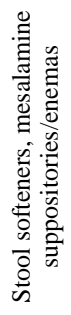 & 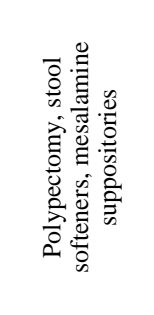 & 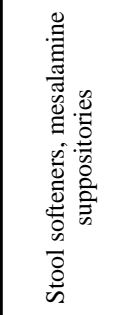 & 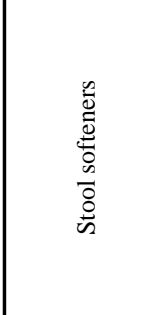 & 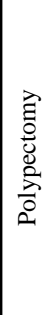 & 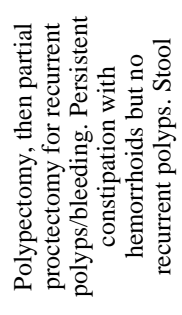 \\
\hline & 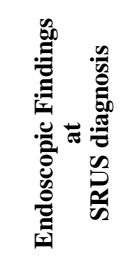 & * & 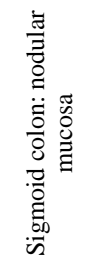 & $\mathbb{z}$ & $\mathbb{z}$ & 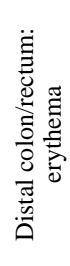 & $\mathbb{z}$ & 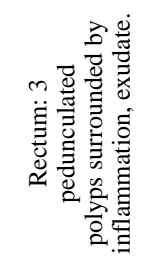 & 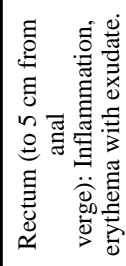 & 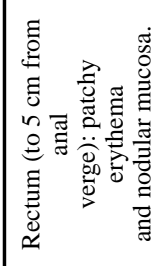 & $\mathbb{z}$ & 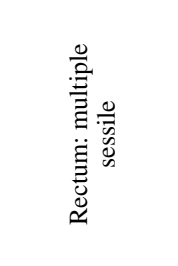 \\
\hline & 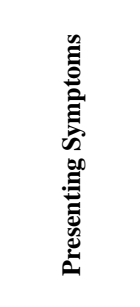 & 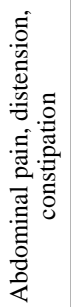 & 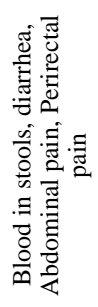 & 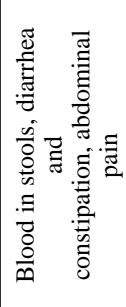 & 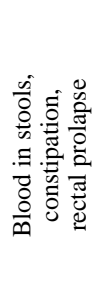 & 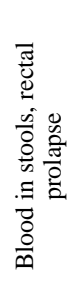 & 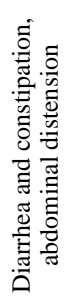 & 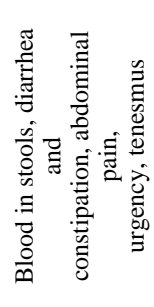 & 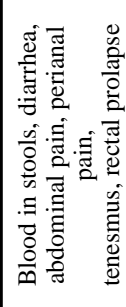 & 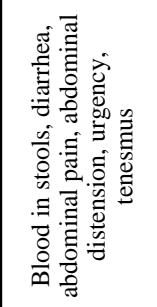 & 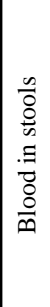 & 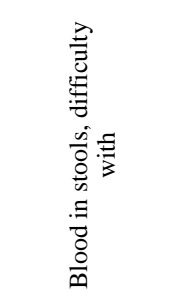 \\
\hline \multirow{4}{*}{ 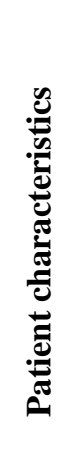 } & 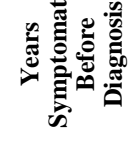 & $\stackrel{\circ}{\circ}$ & $\stackrel{\circ}{-}$ & $\stackrel{\circ}{r}$ & $\mathbb{z}$ & $\stackrel{0}{0}$ & $\stackrel{\leftrightarrow}{i}$ & $\dot{m}$ & ? & $\exists$ & $\stackrel{\circ}{-}$ & $\stackrel{\circ}{+}$ \\
\hline & 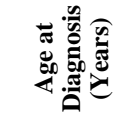 & $\vec{m}$ & $\stackrel{0}{\stackrel{0}{n}}$ & $\begin{array}{l}0 \\
\dot{J}\end{array}$ & $\vec{a}$ & तु & $\vec{i}$ & $\bar{\Xi}$ & $\vec{m}$ & $\grave{n}$ & $\stackrel{m}{ \pm}$ & $\stackrel{\vec{g}}{\mathrm{~g}}$ \\
\hline & U⿺辶ّ & $\Sigma$ & $\Sigma$ & 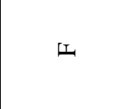 & $\Sigma$ & $\Sigma$ & $\Sigma$ & $\Sigma$ & $\Sigma$ & $\Sigma$ & $\Sigma$ & 山 \\
\hline & 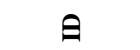 & - & $\sim$ & $m$ & 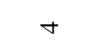 & in & 0 & r & $\infty$ & $a$ & 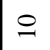 & $=$ \\
\hline
\end{tabular}

J Pediatr Gastroenterol Nutr. Author manuscript; available in PMC 2013 July 23. 


\begin{tabular}{|c|c|c|c|c|}
\hline 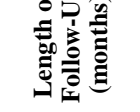 & $\infty$ & 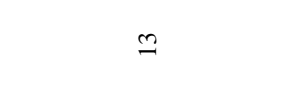 & $\infty$ & $\stackrel{ \pm}{\mathrm{A}}$ \\
\hline 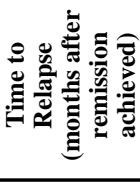 & & $\mathbb{z}$ & & \\
\hline 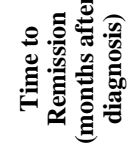 & & $\alpha$ & & \\
\hline 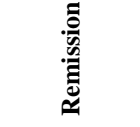 & $\mathrm{z}$ & $\lambda$ & $\overleftrightarrow{z}$ & z \\
\hline 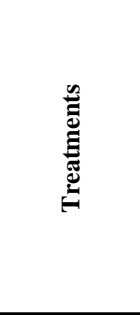 & 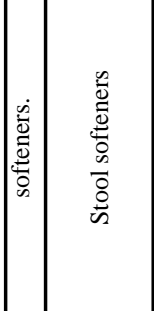 & 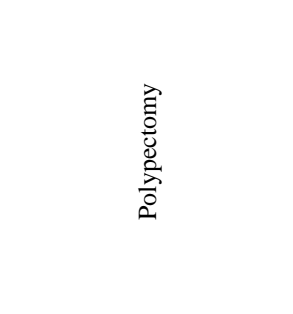 & 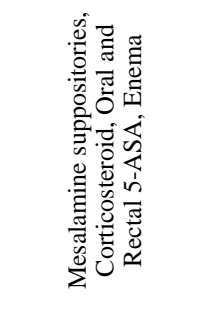 & 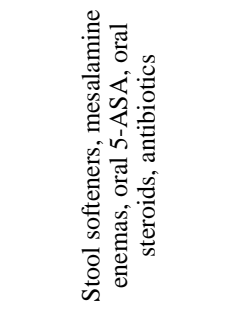 \\
\hline 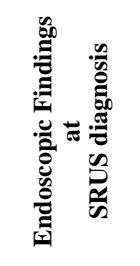 & 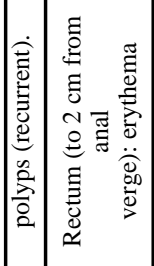 & 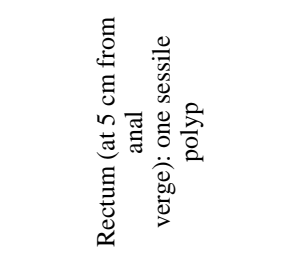 & 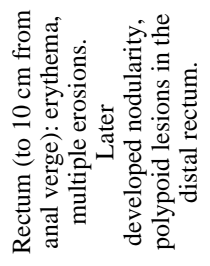 & 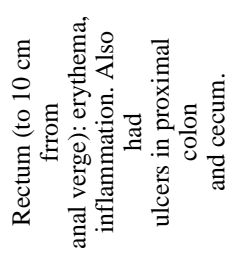 \\
\hline 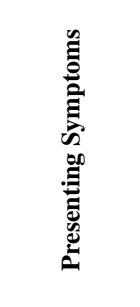 & 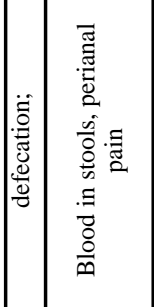 & 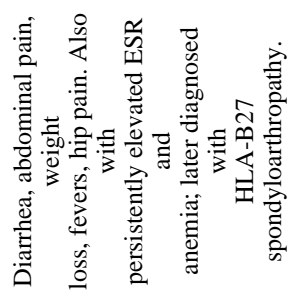 & 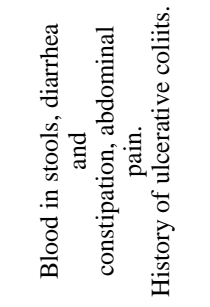 & 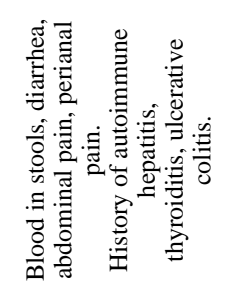 \\
\hline 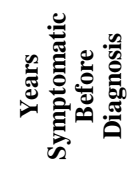 & $\stackrel{m}{?}$ & $\stackrel{+}{-}$ & $\stackrel{乛}{\check{2}}$ & $\hat{i}$ \\
\hline 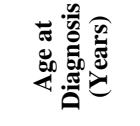 & $\underline{\underline{n}}$ & $\begin{array}{l}n \\
n \\
n\end{array}$ & $\begin{array}{l}0 \\
\stackrel{0}{0}\end{array}$ & $\stackrel{n}{\varrho}$ \\
\hline 苞 & $\Sigma$ & $\Sigma$ & $\Sigma$ & 山 \\
\hline 目 & $\simeq$ & 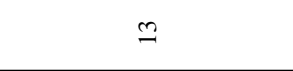 & " & * \\
\hline
\end{tabular}


Table 3

Histologic Findings in Patients at Diagnosis

\begin{tabular}{|l|c|}
\hline & N \\
\hline Muscularization of lamina propria & 14 \\
\hline Thickened muscularis mucosae & 13 \\
\hline Ulceration / Mixed inflammatory infiltrate & 11 \\
\hline Epithelial hyperplastic changes & 10 \\
\hline Misshapen crypts & 8 \\
\hline Vascular ectasia & 8 \\
\hline
\end{tabular}

